\title{
Picking Some News about the Retroviral Vector World from the 9th Symposium of the European Society of Gene Therapy
}

\author{
Claude Bagnis* \\ Département de Thérapie Cellulaire et Génique, Établissement Français du Sang (EFS) Alpes Méditerranée, \\ 149 boulevard Baille 13392 Marseille CEDEX 5, France
}

\begin{abstract}
The 9th symposium of the European Society of Gene Therapy (ESGT) organized by Murat Tuncer (President of the Meeting), Bernd Gansbacher (President of the ESGT), and Meral Ozguc (Secretary of the Meeting) took place in Antalya, South Turkey, on 2-4 November 2001. Although the international political context made difficult the coming of some researchers, this symposium has drawn an interesting picture of the works in progress in Europe and in the Mediterranean area.
\end{abstract}

The emergence of lentiviral vectors, mainly derived from human immunodeficiency virus (HIV) type 1 , at the end of the nineties largely opened the possibility to manipulate cells, which was not considered as classical target cells of the retroviral gene transfer strategy, or which failed to be successfully genetically manipulated using murine leukemia virus (MLV) derived vectors [1]. Taking advantage of the expertise in retroviral vector design raised from the past twenty years, lentiviral backbones have rapidly exhibited common features with the latest MLV derived backbones, allowing numerous works to be carried out to compare MLV derived vectors with lentiviral vectors. Since 1996 with the Naldini's paper describing the possibility to construct and produce HIV-1 derived vectors [2], during the previous ESGT meetings only few oral or poster presentations have reported the use of lentivirus derived vectors as compared to adenoviral vectors or adeno-associated virus (AAV) derived vectors. In 2001, numerous reports highlighted the use of lentiviral vectors and pointed out the interest of these vectors.

\section{TRANSDUCTION EFFICIENCY: STILL THE GOLDEN GOAL, BUT ...}

The testing of new transduction procedures, the interest of additional cis-active sequences such as the central DNA flap sequence in lentiviral vector that improves the transduction of several cell types, the design of new production processes that enable the use of high doses of vectors in vivo, ... increasing the transduction efficiency to reach the $100 \%$ golden goal is a classical duty for people working on gene therapy. But most of the works presented focused on the finding of equilibrium between transduction efficiency and quality of the expression. The combination of the inhibition of the expression of the HIV long terminal repeat in tat-deficient cells and the possibility to use efficient self inactivating backbones that exhibit deletion of the viral promoter in the transduced cell, allowed numerous works to focus on efficient expression strategies. The strength and the expression kinetics of the so-called constitutive promoters can vary from a cell type to another, which makes difficult to ascertain the interest of only one promoter. In spite of this difficulty, the phosphoglycerate kinase (PGK) and the elongation factor 1-alpha (EF1-alpha) gene promoters have been shown to efficiently and for long-term express lentivirally transduced marker genes in various cell types, such as hepatocarcinoma cells, hematopoietic cells, or central nervous system cells, among several constitutive promoters tested including the cytomegalovirus derived promoter, the human alpha antitrypsin gene promoters, and the chimeric CAG promoter. Beside constitutive expression, strong efforts are developed to control the expression of the transgene using tissue-specific promoters in lentiviral vectors. The use of the promoter of the CD4 gene to induce the transcription of the transgene expression unit in CD4 lymphocytes, the insulin gene promoter in hepatocytes, modified versions of the ankyrin gene promoter or the locus control region of the beta-globin gene in erythropoietic cells, an epidermal growth factor responsive promoter in epithelial cells, ... very interesting works have demonstrated the possibility to achieve a selective strong expression in target cells with low level basal expression in non selected cells. Although promising, whether these in vitro models will be successfully transposed to the in vivo approaches remains to be shown. Multiple gene expressing vectors are being tested but-although one work showed an elegant penta-cistronic MLV based vectors, those efficacy and interest remain to be proven-MLV and lentiviral vectors are still dealing with the expression of two genes at maximum with classical coexpression strategies relying on internal ribosomal entry site elements, fusion of the coding sequence of protein, generation 
of cleavable bi-functional precursor proteins, or multiple promoters.

\section{THE IN VIVO TRAP}

Both as a way to control the expression and as a safety feature for in vivo approaches, targeting the binding of the viral particle is still highly considered but with the works only conducted by a few expert groups. The strategies approached with MLV derived vectors to redirect the surface protein (SU) are now being tested with lentiviral particles in vitro with moderate success in terms of transduction efficiency. For example, the targeting of T lymphocytes has been engineered by the use of MLV vectors pseudotyped by SU molecules from HIV, or pseudotyped by the coexpression of the VSV-G envelope protein with a $\mathrm{T}$ cell activating peptide from the anti-CD3 OKT3 monoclonal antibody at the viral particle surface. The natural trapping of viral particles by the liver, the spleen, and to a lower extent by the lung, makes difficult the possibility of achieving efficient transduction by systemic injection of the viral particles and markedly emphasizes the interest of the targeting strategy. But it is to note that the potential trapping of targeted particles by these filter organs remains to be addressed.

The immune reaction makes another in vivo trap for both viral particles and transduced cells. The pseudotyping of lentiviral particles with new surface proteins such as derivatives of the RD114 virus SU, and the use of highly purified vector batches in vivo to prevent from cell mediated immune reaction, demonstrate that the immunological aspects of the lentiviral particle have also to be carefully considered. However, as it has been shown several years ago with MLV vectors, it seems that the moderate immune reaction observed after injection is not sufficient to abolish primary or reiterated gene transfer.

\section{BEYOND THE MARKER GENES}

Most of the strategies using MLV vectors are now being approached by lentiviral vectors with the treatment of hematological genetic diseases such as the Zap-70 deficiency related severe combined immunodeficiency (SCID), the protoporphyria, the chronic granulomatous disease, or the treatment of pathologies that could benefit from the ectopic expression of the transgene such as the ectopic expression of coagulation factors in liver cells for the treatment of hemophilia. For anti-cancer purposes, the genetic manipulation of cells involved in the immune reaction such as dendritic cells is a classical strategy relying on the possibility to express tumor associated antigens, such as Melan A, tyrosinase, gp100, or MAGE-3 to elicit melanoma specific immune responses. Most, if not all, the viral vectors have been tested to transduce dendritic cells and lentiviral vectors can be considered as interesting tools to genetically manipulate these types of cells. It is to note that several reports favor the use of DNA-flap sequence containing lentiviral vectors to genetically manipulate dendritic cells deriving either from hematopoietic progenitors or monocytes. Considering that the interest of the lentiviral vector for the transduction of $\mathrm{T}$ lymphocyte remains to be firmly proven, most of the works relying on genetic manipulation of $\mathrm{T}$ cells, such as transfer of the herpes simplex virus derived thymidine kinase gene to manipulate graft-versus-host disease, or the expression of interleukin-10 to modulate the immune response, did not switch to the lentiviral vector technology, yet.

Lentiviral vectors can be of strong interest to transduce nondividing cells in the treatment of some pathologies usually approached by adenoviral vectors or AAV derived vectors. For example, lentiviral gene transfer into neuronal cells in vivo has been reported for the study or the treatment of diseases affecting the central nervous system, with the transfer of the Huntington protein to produce a model of Huntington disease, or the lentiviral transfer of the GDNF for the treatment of Parkinson's disease.

\section{FROM THE BENCH TO THE BEDSIDE: WAITING FOR SOME INDUSTRIAL IMPROVEMENTS TO MAKE THE CHOICE BETWEEN MLV AND LENTIVIRAL VECTORS}

The main limitations of the lentiviral vector strategy remain the large-scale production and clinical development. These two issues, which still have to be addressed, keep attracting some strategies using MLV derived vectors for some applications.

The design of large-scale production of lentiviral vectors is in process. However, information about this technological development is difficult to obtain from people or companies involved in such projects, mainly due to confidentiality agreement barriers. Safety concerns regarding lentiviral vectors have not been firmly discussed but was present as an ulterior motive in the presentations dealing with this strategy. It is unfortunate to notice that discussions on the development of replication competent virus (RCV) assay to detect fully replication competent contaminants or intermediate recombinant genomes that could favor RCV occurrence, were absent from the presented works.

Beyond the technological problems, some cell types such as proliferating $\mathrm{T}$ lymphocytes can be efficiently transduced and selected using either lentiviral or MLV vectors. In addition, the gene therapy treatment of some pathologies, such as the gamma $\mathrm{c}$ chain deficiency SCID, a priori, does not require the use of lentiviral vectors. In some instances, such considerations favor the use of MLV vectors that is an already clinically available concept.

\section{FROM MLV VECTORS TO LENTIVIRAL VECTORS: AN IN-BETWEEN PERIOD?}

The evolution of MLV vector seems to pass by a plateau. Conversely, according to the large area of possibilities still opened for the design and the in vitro and in vivo use of lentiviral vectors, strong evolutions of the lentiviral vector strategy have to be envisioned in the near future. Lentiviral vectors can be considered now as very interesting tools to focus on. But MLV derived vectors are not becoming oldfashioned tools. The information feedback we can expect 
from any progress in this field will help us to improve previously approached gene transfer vectors or will allow us to reconsider the way to use them. Beyond their intrinsic properties, lentiviral vectors will also help us to make a step further in gene transfer technology using MLV vectors or other tools.

To allow this overall improvement in gene therapy, whether the use of lentiviral vectors can impact the therapeutical achievement expected in gene therapy has now to be demonstrated. A first clinical trial using lentiviral vectors has been discussed at the Recombinant DNA Advisory Committee (RAC) session on 2001 [3]. The way is opened.

\section{REFERENCES}

[1] Vigna E, Naldini L. Lentiviral vectors: excellent tools for experimental gene transfer and promising candidates for gene therapy. J Gene Med. 2000;2:308-316.

[2] Naldini L, Blomer U, Gage FH, Trono D, Verma IM. Efficient transfer, integration, and sustained long-term expression of the transgene in adult rat brains injected with a lentiviral vector. Proc Natl Acad Sci USA. 1996;93:11382-11388.

[3] Podsakoff GM. Lentiviral vectors approach the clinic but fall back: national institutes of health recombinant DNA advisory committee review of a first clinical protocol for use of a lentiviral vector. Mol Ther. 2001;4:282-283.

${ }^{*}$ E-mail: claude .bagnis@ef s. sante.fr

Fax: +33 4911895 98; Tel: +33491189500 

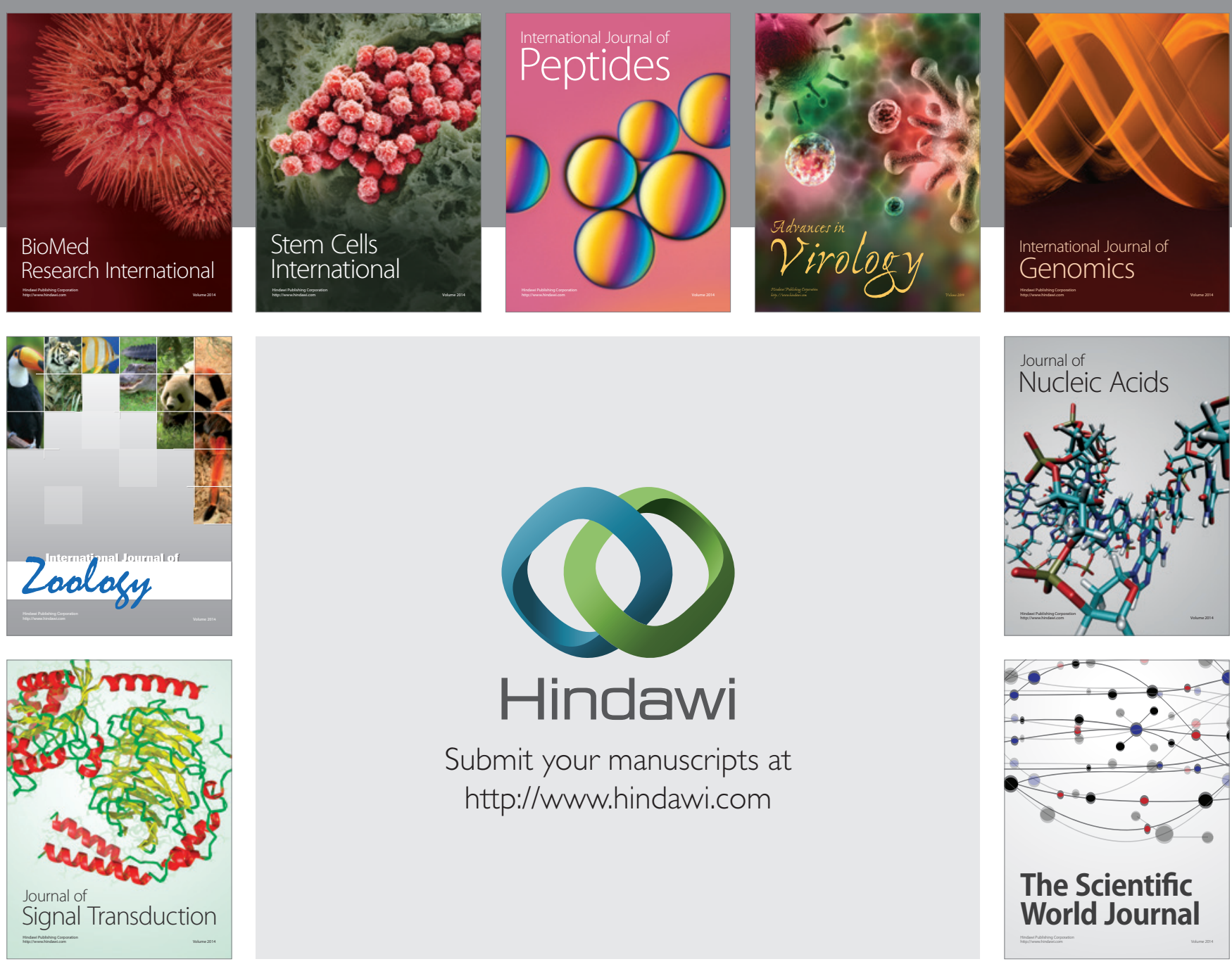

Submit your manuscripts at

http://www.hindawi.com
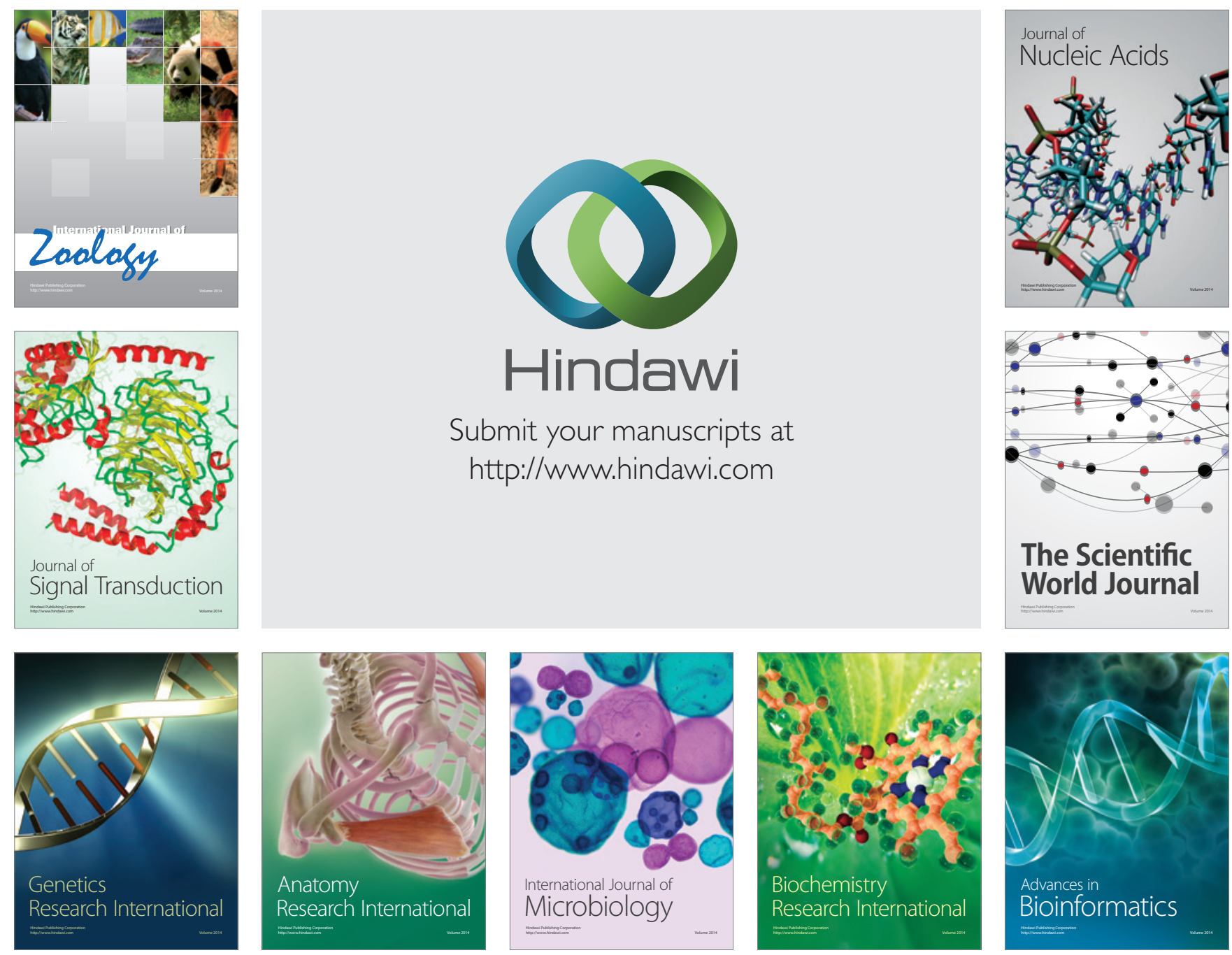

The Scientific World Journal
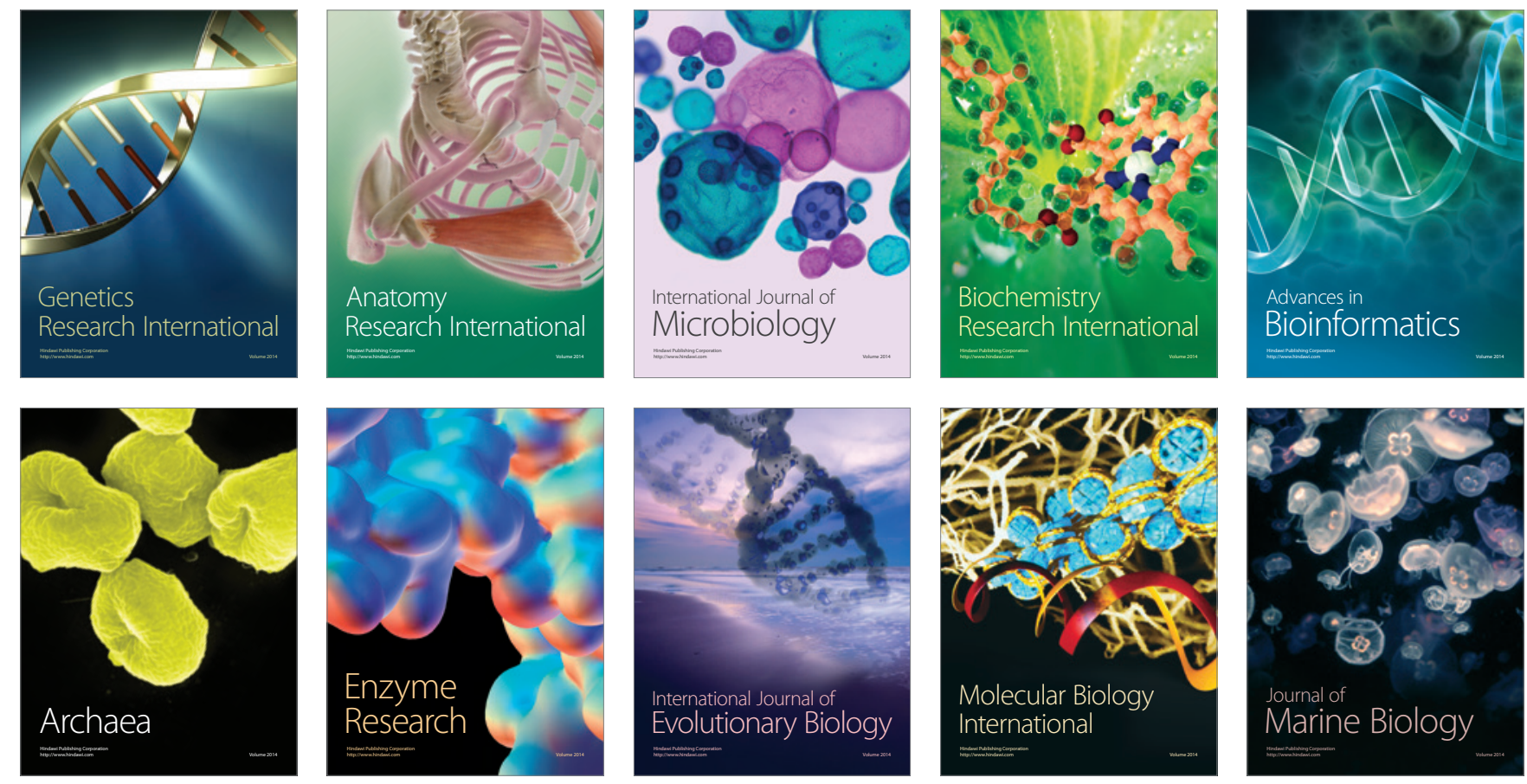